\title{
Concurrent Design and Analysis of the Navigator Wearable Computer System: The Thermal Perspective
}

\author{
Cristina H. Amon, Jay S. Nigen, Daniel P. Siewiorek, Asim Smailagic, John Stivoric \\ The Engineering Design Research Center \\ Department of Mechanical Engineering, and \\ School of Computer Science \\ Carnegie Mellon University \\ Pittsburgh, PA 15213
}

\begin{abstract}
This paper describes the concurrent design of a wearable computer, called the Navigator, developed and built at Carnegie Mellon University in a multidesigner, multidomain environment. The design effort for the Navigator involved nineteen designers, representing the electronics, mechanical, industrial, software and human-computer interaction disciplines. The evolution of the multidisciplinary design is described, with particular emphasis placed upon the role of the thermal design group in the overall design process. Furthermore, the particular challenges associated with the concurrent thermal management of wearable computer systems are outlined.
\end{abstract}

\section{Introduction}

Consumer desire for increased computational functionality has resulted in the development of portable computers. Portable computers, as exemplified by today's notebook machines, required technical innovations in both hardware and software to overcome the challenges presented by the associated size, weight, and power consumption constraints. Additional constraints, which are market imposed, result from the highly competitive nature of the computer industry, necessitating shorter time-tomarket, higher levels of quality, and lower cost. The satisfaction of these competition-driven constraints requires the achievement of domain specific objectives, such as clock speed, without the violation of any seemingly unrelated global constraints, such as reliability. Additional design iterations, required to correct any unresolved constraints or unanticipated flaws, lengthens the development cycle. Competition has reduced the acceptable development cycle to less then twelve months which, eliminates the time needed for additional design iterations. Therefore, the participation of the entire design team is required to insure that a discipline-specific modification does not adversely affect other performance objectives.

The recognition of the multidiscipline nature of design has fostered interest in concurrent design, through which the concerns of a design team are accounted for in a collaborative effort. This cooperative approach improves communication between designers, with the intent that resultant products are error free, and as such, correct-by-design. These products satisfy both the desire for higher quality and shorter development cycles.

This paper describes the concurrent design of a wearable computer, called the Navigator. The Navigator is the third generation of wearable computers developed and manufactured at Carnegie Mellon University (CMU) (Akella et al., 1992). The Navigator allows hands-off operation with a speaker-independent, 200 word vocabulary continuous speech recognition system for input and a head worn display for output (Smailagic and Siewiorek, 1993). It provides wireless communication with a remote site, as well as global position sensing, so that information relevant to a location can be displayed. In some applications, such as manufacturing, we envision sensing the direction which the user is viewing, so that information specific to that viewing angle, such as an assembly step, can be dispiayed. Position sensing is currently used for the Navigator's application of self-guided navigation around the CMU campus. Likewise, different applications would require a variety of access rates to remote databases, resulting in a range of remote communication options from low data rate cellular phones over long distances to high bandwidth radio frequency local area networks.

The initial specifications for the Navigator included: Functionality

- Hands-off operation

- Speaker independent, continuous speech recognition

- Capability to display text and graphics

- Miniature, light-weight, heads worn display

- On board database of information and maps

- Differential Global Position Sensing (GPS)

- Modem/wireless communication with re mote site

Performance

- Weigh less than 10 pounds

- At least two-hour battery lifetime before recharging

- Position sensing accurate to 5 meters

- $80-90 \%$ accuracy in speech recognition

- Screen refresh should not distract the user

- Cost less than $\$ 4500.00$ per unit

A multi-disciplinary approach brings together personnel with vastly different expertise. The following disciplines are included in the design process:

- Electronics: electronic components, electronic interfacing, power supply 
- Mechanical: ergonomics, housing, thermal manage ment, mechanical fixtures

- Software: operating systems and support software, such as user interface tools, libraries for position sensing and telecommunications, hardware drivers

- Human-Computer Interaction (HCI): speech and user interface (UI)

The design disciplines define the groups for the initial personnel organization. Subsequently, personnel are reassigned into interdisciplinary design teams as the various subsystems are identified and refined.

\section{Conceptual Design}

There are many possible solutions to a design problem. During the conceptual design phase of a project, designers are encouraged to actively communicate ideas and information. By generating and discussing multiple design alternatives, designers can synthesize a solution that will most effectively support the initial design requirements and satisfy user needs.

Within this initial stage, designers establish a common vision of the end product. This vision provides a consistent set of design goals for all disciplines to maintain throughout the product development cycle. Based on this team vision, the Navigator resulting product concept is a modular design that enables the system to be adaptable to a variety configurations and applications. Both physically and electronically, potential users of the Navigator system can individually tailor the unit according to application-specific needs. In addition, because the modules are designated/subdivided by the tasks that they perform, updating or repairing the system can occur simply by unplugging and replacing the appropriate module. These tasks include information processing, visual display, speech recognition, position sensing, telecommunications and power.

During the conceptualization stage of the design evolution, the thermal designer participates by insuring that all team members are cognizant of cooling issues. By doing so, the design team can focus on concepts that satisfy their own objectives, while also minimizing heat production and accommodating any necessary cooling strategies. The requirement for outdoor usage virtually mandates the Navigator to be a closed, ventless system. Therefore, heat dissipation needs to be kept to as low a level as possible. The envisioned modular approach is advantageous from the thermal perspective, for it subdivides the total power output and increases the surface area through which heat can be dissipated. The desire for extended battery life incorporates power minimization as a shared objective between the electronics and thermal designers.

After the basic parameters are bound and an acceptable concept for the design specified, concurrent design is initiated. First, the concurrent design framework used by the Navigator design team will be outlined, stage by stage. A synopsis of the status of the designers will be included for each stage. Following this, each design stage will be expanded from the perspective of the thermal designer. The specific issues and interactions related to the thermal designer are described so that, the role of the thermal designer in the concurrent development of the Navigator is depicted.

\section{Concurrent Design Procedure}

Throughout the design of the three generations of wearable computer systems, a Concurrent Engineering Methodology has evolved (Siewiorek et al., 1993). The goal of the methodology is to allow as much concurrency as possible during the design process. Concurrency is sought in both time and resources. The entire design cycle is divided into phases, in which activities proceed in parallel, but are synchronized at phase boundaries. Furthermore, the thermal design and analysis tools used in concurrent design must increase in complexity with the evolution of the product and reduction of the design space, thereby, satisfying both the accuracy and time constraints associated with a particular design stage (Nigen and Amon, 1992).

Although the availability of inexpensive, fast computers permits numerical prediction of complex thermo-fluid phenomenla in electronic systems not previously possible, (Amon, 1992; Nigen and Amon, 1993) the thermal analysis of many such phenomena still remains intractable in a design environment. This is especially true in concurrent design because of the stringent time constraints associated with the early design stages. In particular, the large design space associated with initial design stages prohibits direct simulation of all permutations of a design (Yesilyurt and Patera, 1993). To address this difficulty, a concurrent thermal design methodology was proposed that utilizes successive model refinement (Nigen and Amon, 1992). Surrogate models are employed in the early design stages to reduce the space, after which, successively more complete and accurate models are employed. The model evolution culminates with a conjugate conduction/convection direct numerical simulation of the finalized design.

The generic design process is broken down into six sequential phases:

\section{Technology Survey}

Alternative technologies are identified for each subsystem, evaluated in isolation from other subsystems, and compared using a Model Feature matrix. A Model Feature Matrix is a concise summary of the technological alternatives that reflects the attributes and characteristics identified as relevant during the project team meetings. It is based on information gathered mostly from product literature and is used to identify primary and backup alternatives for each of the subsystems.

\section{System Architecture Specification}

This phase attempts to integrate the results of the technology survey phase to produce the first concept of the total system. Interactions between subsystems are identified and inconsistencies between subsystem alternatives are detected. Interactions between subsystems are summarized in a Design Dependency Matrix, which is a chart that identifies all dependencies on other projects and subgroups, and whether the dependencies have been resolved or remain open issues. These dependencies identify communication points for subsequent phases of the project. The primary technology alternative for each subsystem is refined to eliminate inconsistencies and a preliminary architecture for the total system is specified.

\section{Subsystem Specification}

Both firm and probable design decisions are identified for each subsystem based on these design decisions. Interactions between each subsystem are specified completely so that other 
subsystem designers can continue into the detailed design phase. Subsystem specifications are integrated into a complete Product Design Specification, which defines two levels of specifications, subsystem and total system, and emphasizes interface behavior. This phase represents the last exploratory phase of the design process.

\section{Detailed Design}

A detailed design of each subsystem is performed, with particular attention to maintaining the interface specifications as defined in the Product Design Specification. The technology selected for each subsystem is acquired and analyzed in terms of functionality and performance. Analysis of the technology may necessitate changes in the subsystem specifications which are then communicated to the relevant groups. Regular design reviews are held with group liaisons to ensure that interface specifications are not violated. A Task Dependency Graph, which is a refinement of the Design Dependency Matrix, is used to identify individual design decisions that affect more than one group, so that changes can be rapidly propagated. The Task Dependency Graph is also used to explicitly identify interfaces between groups and potentially critical paths that might require additional resources.

\section{Implementation}

After the detailed design is completed, each group implements its subsystem using the acquired technology. As implementation progresses, the subsystems are demonstrated at various stages of development. A check list of open issues and action items is used to highlight remaining design dependencies and schedule their resolution.

\section{System Integration}

Each subsystem is individually tested and then integrated into the final system. An Integration Tree is used to sequence the merging of subsystems along interfaces (Siewiorek et al, 1993).

Next, the evolution of each design phase of the Navigator wearable computer is presented and the role of the thermal designer is described.

\section{Concurrent Design of the Navigator}

\section{Technology Survey}

After reviewing the preexisting mobile systems, the project team brainstormed alternatives for the Navigator wearable computer. Issues for evaluating design alternatives, such as cost, weight and development time, were also identified. The target application was navigation around the CMU campus. To concurrently explore each of the technology alternatives, the project team was split into four generic subgroups: electronics, mechanical design/housing, system software, and applications. The technology survey phase was conducted independently by each group. Literature was surveyed, vendors contacted, and local experts consulted to identify potential options. The findings in the Technology Survey were reported as Model Feature Matrices, wherein, alternative models were listed and compared by the evaluation issues initially identified. Results of the Technology Survey phase were communicated to the project team and reports, including product specification sheets, were distributed to all team members.
From the thermal design perspective, the combination of portability and concurrency presents significant challenges. The premium placed upon power consumption, size, and weight discounts many of the previously popular cooling techniques. In the case of the Navigator, the situation is further complicated by the necessity of a ventless housing. As with many portable systems, a closed housing is desirable because the electronics have to be shielded from environmental contaminants and moisture. Cooling strategies for portable computers are further constrained by the desire of minimizing parasitic power consumption, such as that required to drive a fan. Therefore, a more integrated approach is required, whereby, the housing is used as the heat sink and heat flow paths are carefully planned to insure adequate cooling for both reliability and comfort considerations. Furthermore, thermally-sensitive components, such as the CPU and memory boards, must be carefully positioned within the housing to insure adequate heat removal.

The initial thermal design concept calls for the incorporation of conductive fins (Figure 1) that would directly connect heat producing components to the inside surface of the housing. The thermal performance of this approach suffers from the high contact resistances, characteristic of solid-to-solid interfaces, which may become a limiting factor of the thermal performance, as well as temperature gradients between the package and housing surfaces. An alternative approach was described by Oktay (1993), which utilizes flexible heat pipes with variable crosssectional area to connect heat producing components with the housing. Heat pipes offer the advantage of transferring high flux over long distances with minimal temperature drops, but retain the difficulties associated with contact resistances. Furthermore, flexible heat pipes are far more expensive and, as such, would only be incorporated if the conductive fin approach proved ineffective.

\section{System Architecture Specification}

The primary alternatives for each group were presented in detail during team meetings. Subsystem interactions and dependencies were identified and used to indicate which design groups to notify if design decisions were made or modified. Compatible design alternatives were labeled resolved (i.e., the disk drive and the processor interfaced to the same bus, the operating system had a software device driver for the disk drive, etc.), while other dependencies were labeled unresolved and required further refinement in the next phase.

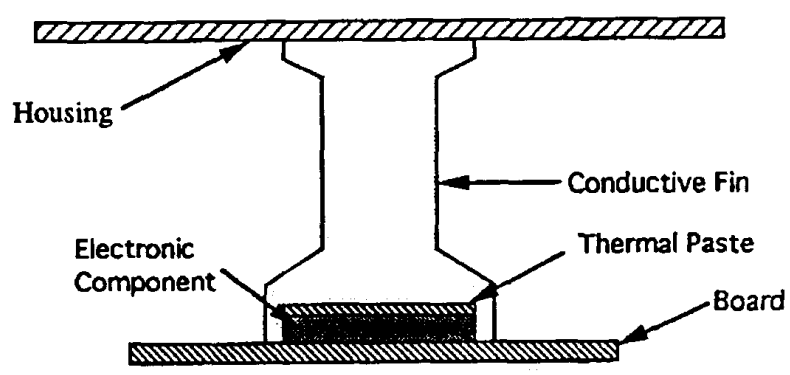

Figure 1. Schematic of a conductive fin 
A new group was formed to focus on speech recognition since this appeared to be the subsystem which was the least well understood and developed. Sphinx 1 (Li et al., 1989) was selected as the primary alternative for speech recognition and Mach (Rashid et al., 1989) was selected as the primary multitasking operating system, both due to the availability of local experts. The Intel $80 \times 86$ processor family was the processor of choice for Mach. Since the 80386 had the highest performance and was the lowest power consuming $80 \times 86$ chip at the time of the project, design alternatives were narrowed to low power consuming boards based on the 80386 . The only satisfactory alternative for display was found to be the Private Eye (Becker, 1992) and it became the default. Other dependencies could not be resolved without further study and resolved and unresolved design alternatives were discussed during the group presentations.

The thermal designer determined an approximate ratio between heat dissipation and required housing surface area through the use of Figures of Merit, as shown in Figure 2 (BarCohen, 1992). However, this lumped formulation requires the assumption of a safety factor to account for the deviation between the average temperature of the system and the junction temperatures of the heat producing components. This can be especially significant in electronic systems because of the large degree of material variation and concentration of heat generation. The information provided by this analysis indicated the approximate number of housing modules required for the complete Navigator system. Additionally, through the use of surrogate one-dimensional models, proposed housing materials and thicknesses are evaluated relative to thermal performance criterion.

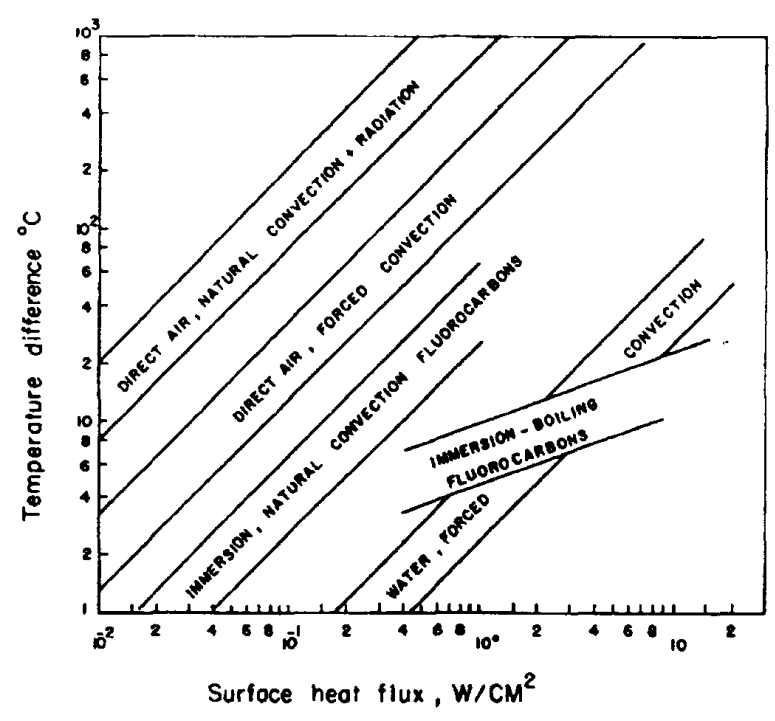

Figure 2. Temperature rise versus surface heat flux for different cooling techniques

The initial thermal design incorporated an integrated heat sink with conductive fins connecting the heat producing components to the housing surface. Components along the outside perimeter of boards could be directly attached, but components within the central regions of the boards would require an alternate connection technique. The heat sink was composed of aluminum and was directly attached to the inside surface of the housing. An additional constraint associated with the heat sink was that no region of the housing outside surface should reach temperatures uncomfortable to the human touch.

\section{Subsystem Specification}

The goal of this phase is to produce an interface specification between subsystems so that the detailed design and implementation can proceed concurrently between groups. Subsystem specification represents the last exploratory phase of the design and combines both bottom-up and top-down features. The topdown is based on a specification of the total system, from which work proceeds on the appropriate subsystems. For the bottomup approach, each subgroup provided a subsystem specification with special emphasis on how the subsystem will interact with the complete system. The interfaces were specified in sufficient detail for other groups to continue into the next design phase. The subsystem specifications were combined into a Product Design Specification for the entire system.

After the electrical hardware design team had selected the various components and boards, a solid model and elemental discretization is constructed in order to perform heat conduction simulations. Difficulty was encountered in the determination of material properties and power dissipation for each component. As an example, the in-house designed board was expected, based upon manufacturer specifications, to dissipate 22.18 watts. However, experimental measurements indicated that only 7.50 watts are dissipated, corresponding to $33.7 \%$ of the published values. Further complications result from the necessity of balancing computational viability, associated with processing and memory limitations, with spatial resolution.

The Navigator is a multi-board, real-world system containing many components. To satisfy computational resource constraints, most of the components were represented by a single element, which requires modeling the packages as uniformly heat generating, single-material components. Krueger and BarCohen (1992) have suggested a procedure whereby accurate thermal performance can still be obtained through the use of thermal influence coefficients. In this study, volume-weighted averages were used to model package material properties and contact resistances were modeled by placing a thin plane of interface elements between each package and its associated board. The thermal conductivity of these interface elements was adjusted to match temperature drops commensurate with those found experimentally (Yovanovich and Antonetti, 1988). The resulting computational spectral-element mesh for the thermofluid simulations, (Patera, 1984; Amon and Mikic, 1991) displayed in Figure 3, consists of 660 macro-elements, each containing 125 degrees of freedom.

The first level of numerical predications is based on constant surface temperature boundary conditions. Under these conditions, the maximum temperature rise $(\Delta T)$ is obtained for the components, which provides qualitative information as to the location of those exhibiting high operating temperatures. It is essential for such information to be obtained early in the design 


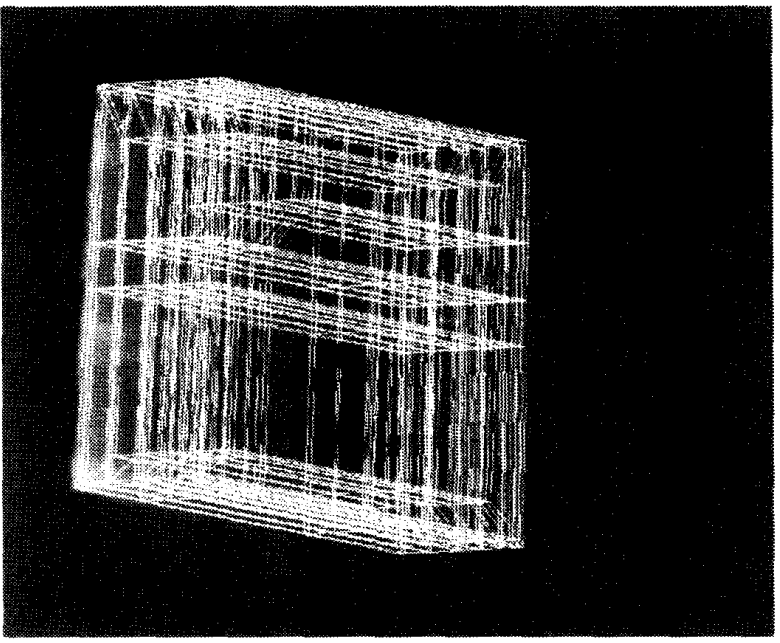

Figure 3. Spectral-element discretization for the heat conduction simulations

process to allow for advantageous component placement relative to the attachment of conductive fins. Additionally, component placement can sometimes be rearranged to separate those that dissipate the largest amount of heat, but this must be accomplished without adversely affecting the electronic performance of the artifact. The mechanical design team also incorporated the available thermal-performance data, along with manufacturing and industrial criteria, to determine board and component orientations.

\begin{tabular}{|lll|}
\hline Component & Description & $\Delta \mathrm{T}\left({ }^{\circ} \mathrm{C}\right)$ \\
\hline $\begin{array}{l}\text { ADM 8038SX-25 } \\
\text { ULSI 80386SX }\end{array}$ & $\begin{array}{l}\text { processor } \\
\text { coprocessor }\end{array}$ & $\begin{array}{l}0.0036 \\
0.0042\end{array}$ \\
\hline SIMS & Dynamic RAM & 0.0338 \\
\hline LM7905 & Voltage Regulator & 0.1305 \\
LM340 & Voltage Regulator & 0.1305 \\
\hline Hard Drive & \multicolumn{1}{|c}{} & 0.0023 \\
\hline LM350T & Voltage Regulator & 0.0879 \\
AOX VCGA & VGA & 0.0084 \\
\hline
\end{tabular}

Table 1a. Maximum temperature rise per component using constant surface temperature boundary conditions.

The results of these numerical simulations (Table 1a) must be interpreted considering that the physically realized convective boundary conditions would be far lower than an infinite heat transfer coefficient, which corresponds to constant temperature boundary conditions. Seemingly small temperature increases above the prescribed surface values could correspond to significant temperature rises in the real system. Therefore, both LM7905 and LM340 voltage regulators require direct attach- ment to the heat sink. These componcnts are located on the outside perimeter of their respective boards, which simplifies the design of the conductive fins.

\section{Detailed Design}

A subdivision and reassignment of personnel allowed for a focusing of resources on a maximally concurrent set of tasks. The subsystem interface behavior became the implied guideline between groups. Task Dependency Graphs were used to explicitly identify interfaces between groups and identify potential critical paths that required more human effort and resources.

The current implementation of the Sphinx 1 speech recognition algorithm does not run in real time, and hence, is unable to deal with a continuous stream of speech input. If a buffer is used to hold the input data, this implies that it will be filled at a faster rate than the data is interpreted by the algorithm. To support an input buffer of finite size, a limit on the maximum duration of continuous speech input is necessary. Likewise, a mechanism is required for detecting the onset and discontinuation of speech, as well as to enable and disable input stream buffering, respectively. Such a mechanism must run in real time and can be implemented in either hardware or software. Thus, a custom speech detection circuit was conceived and implemented as part of the Navigator design. This new board added to the power and housing requirements.

A numerical heat conduction simulation, using constant temperature boundary conditions, was conducted for the custom speech detection board. The resulting temperature rise for the hottest components on this board are indicated in Table $1 \mathrm{~b}$. Both the +5 and -5 volt power supplies are hot enough to require direct attachment with conductive fins and are added to list of potentially thermally problematic components.

\begin{tabular}{|lcc|}
\hline Component & Description & $\Delta \mathrm{T}\left({ }^{\circ} \mathrm{C}\right)$ \\
\hline+5 volt Power Supply & - & 0.2116 \\
-5 volt Power Supply & - & 0.0485 \\
\hline
\end{tabular}

Table 1b. Maximum temperature rise per component on the speech recognition board using constant surface temperature boundary conditions.

For this stage, the thermal designer conducted two types of numerical analyses. The first used the same computational mesh as in the Subsystem Specification stage, except that the hottest components were attached to the heat sink. Special interface elements were placed between the top surface of these components and their associated conductive fins. The magnitude of contact resistance, associated with the decrease of thermal conductivity at any solid-to-solid interfaces, is varied to determine the amount of interface management that provides satisfactory operating temperatures. This is especially important because interface management techniques can range from 
simple approaches, such as thermally conductive glues, greases and pastes to relatively complex approaches, as exemplified by the use of springs and pistons in the IBM TCM (Chu and Simons, 1993). Therefore, through the variation of the conductive resistance within the interface elements, an adequate approach could be determined for each component. The temperatures predicted by these simulations indicate that satisfactory cooling is obtained with the interface element conductivities equal to ten times that of air, implying that a simple interface management technique, such as thermally conductive paste, is adequate.

An additional difference between the previous simulations lies in the imposition of convective boundary conditions rather than that corresponding to constant surface temperature. Difficulty exists in selecting the proper correlation for configurations that differ considerably from the idealized situation of flat plates with uniform heat flux or surface temperature. A variety of correlations were used, ranging from parallel flat plates to those for natural convection in enclosures (Moffat and Ortega, 1988; Peterson and Ortega, 1990). For the later configuration, scaling analysis was used as indicated by Bejan (1984) to estimate the convective flow patterns from which, the proper correlation could be discerned. However, it was found that the predicted temperatures were not substantially different and, therefore, the most conservative values for the heat transfer coefficient were used. The results for the hottest components are displayed in Table 2 and graphically for the entire system in Figure 4 for an ambient temperature of $25^{\circ} \mathrm{C}$. Both of these representations indicate that adequate cooling is achieved through the use of the integrated heat sink. Furthermore, the components that are the least sensitive to high operating temperatures, namely the voltage regulators and power supplies, exhibit the highest temperatures in the system.

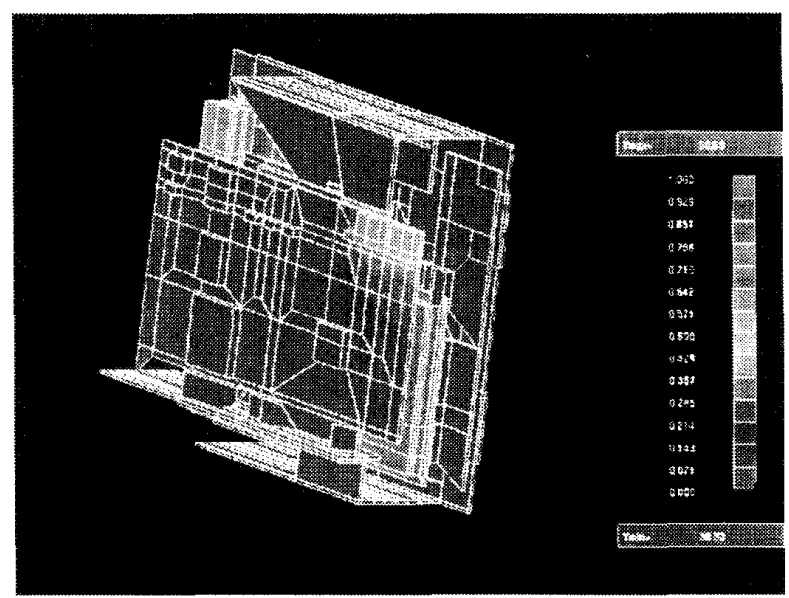

Figure 4. Surface temperatures $\left({ }^{\circ} \mathrm{C}\right)$ predicted by conduction simulations with convective boundary condition

\begin{tabular}{|ll|}
\hline Component & $\Delta \mathrm{T}\left({ }^{\circ} \mathrm{C}\right)$ \\
\hline ADM 8038SX-25 & 8.9861 \\
ULSI 80386SX & 5.8472 \\
\hline SIMS & 10.3846 \\
\hline +5 volt Power Supply & 23.3875 \\
-5 volt Power Supply & 14.9864 \\
\hline Hard Drive & 9.0633 \\
\hline LM350T & 14.2569 \\
AOX VCGA & 6.0659 \\
\hline LM7905 & 9.4834 \\
LM340 & 10.4477 \\
\hline
\end{tabular}

Table 2. Maximum temperature rise per component using a specified heat transfer coefficient as boundary conditions.

The second numerical prediction of thermal performance was based on a different computational mesh that incorporated the air gaps between the boards and housing, as well as the housing material. This mesh was also used in the next stage to conduct a conjugate conduction/convection simulation. Owing to the large computational requirements required for a conjugate study of the Navigator, each board/component level was modeled as a plane. The regions of each plane corresponding to the location of heat producing components are specified to uniformly generate heat. The purpose of this simulation is to determine the surface temperatures of the housing and to further check the basic indications provided by the preceding simulations. Furthermore, the very close proximity (less than $0.5 \mathrm{~cm}$ ) of many of the boards, coupled with the surface height variation caused by the components, would serve to reduce convective velocities. Therefore, a conduction-only model provides a conservative estimate of thermal performance. The resulting temperature field, shown in Figure 5, indicates that acceptable operating temperatures are obtained, and that the exterior of the housing exhibits satisfactory temperatures from a comfort perspective. Since the Navigator is a portable system, it is important that the outside surface of the housing exhibit relatively low temperatures.

\section{Implementation}

As each design group implements their subsystem, an evolving list of open issues, action items, and responsible designers is identified to indicate the remaining design dependencies and their resolution. Furthermore, a progression of logical milestones is monitored, including demonstrations and written reports.

In this phase, the development of the speech subsystem was completed and the code ported to the open-air system. The open-air system is comprised of the Navigator's electronic components, interconnected on a desktop, and was used to verify operations on the final hardware platform. A microphone and 


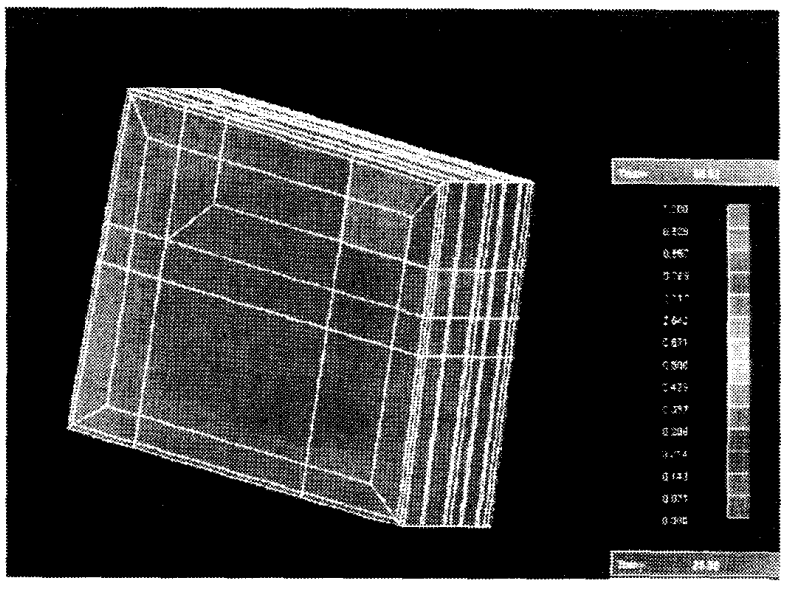

Figure 5. Surface temperatures $\left({ }^{\circ} \mathrm{C}\right)$ of the Navigator housing for conjugate conduction/convection.

Analog to Digital Converter (A/D) were added to the open-air system, as well as software drivers, supplied with the A/D board, to write audio input samples to a RAM disk. An interrupt from an audio level detector triggers data collection and a serial line driver is used to send data between DOS and Mach computers.

The thermal designer carried out a conjugate conduction/ convection simulation during this stage. As this is the last stage before system integration, it is essential that any possible thermal problems be determined. The previous numerical predictions indicated that the heat sink/conductive fin combination proved adequate in maintaining acceptable operating temperatures. Therefore, the thermal designer related this information to the entire product development team.

The Navigator electronics is composed of mostly off-shelf components that would require mounting fixtures within the housing. However, the housing was manufactured using pressure forming which disallows integration of mounting fixtures. Furthermore, the operating environment for the Navigator necessitates rigidity for integrity. Therefore, the heat sink was modified such that it incorporates fixtures for the boards and components, and increases the rigidity of the housing. This design decision required a collaborative effort among all of the members of the mechanical design team.

\section{System Integration}

The final design phase consists of joining all the subsystems together and implementing their interfaces. An Integration Tree is used to sequence the integration steps and set deadlines for the next step within this phase.

Speech was integrated with the campus application software. An object model was defined for the campus tour application, which included the capability to provide information on individuals, buildings, as well as generate paths to different campus locations. A grammar was generated that matched the user interface and could both assist novice users and provide shortcuts for experienced users.

During this stage, the Navigator was experimentally tested by the thermal designer. As with all numerical investigations/ designs, experimental verification is required to insure the accuracy of the modeling procedure. Thermocouples are used to measure the surface temperatures of the components. The operating temperatures measured experimentally are within the range of temperatures predicted numerically, as displayed in Table 3, and are not excessive. Furthermore, the +5 volt power source is the hottest component within the system.

\begin{tabular}{|lccc|}
\hline Component & Description & $\Delta \mathrm{T}_{\text {exp }}\left({ }^{\circ} \mathrm{C}\right)$ & $\Delta \mathrm{T}_{\text {num }}\left({ }^{\circ} \mathrm{C}\right)$ \\
\hline ADM 8038SX-25 & processor & 36.5 & 34.0 \\
\hline SIMS & Dynamic RAM & 29.5 & 35.4 \\
\hline $\begin{array}{l}+5 \text { volt Power } \\
\text { Supply }\end{array}$ & - & 49.5 & 48.4 \\
\hline Hard Drive & - & & \\
\hline LM350T & Voltage Regulator & 36.0 & 39.0 \\
AOX VCGA & VGA & 35.0 & 31.1 \\
\hline
\end{tabular}

Table 3. Comparison of experimentally measured $\left(\Delta \mathrm{T}_{\mathrm{exp}}\right)$ and numerically predicted $\left(\Delta \mathrm{T}_{\text {num }}\right)$ operating temperatures.

As the system was assembled, modifications were made to the conductive fins to conform to attachment specifications, as well as to accommodate pins that penetrate through adjacent boards. However, it should be emphasized that no additional effort was needed to correct errors associated with miscommunications during the Navigator design.

The completed Navigator wearable computer is shown in Figures 6 and 7, displaying the Navigator electronics and subsystems prior to assembly, and the complete system including the housing, respectively. The timeline of the Navigator project with one overview of the major events in the conception, design, and implementation is shown in Figure 8.

\section{Summary of Resource Allocation}

The designers of the Navigator wearable computer were dynamically reallocated four times during the design process as new tasks were identified. Two development platforms were utilized. A PC platform allowed initial software development. A bench-top, open-air configuration of the final hardware components augmented by Ethernet interface, console monitor, and keyboard allowed software integration prior to availability of the final packaged wearable system. An extensive array of communication techniques were used including group presentations, progress reviews, and group meetings. The communication techniques and resources were dynamically modified throughout a six stage design process described earlier.

\section{Personnel}

The Navigator was initially decomposed into subsystems corresponding to the various disciplines involved. The design 


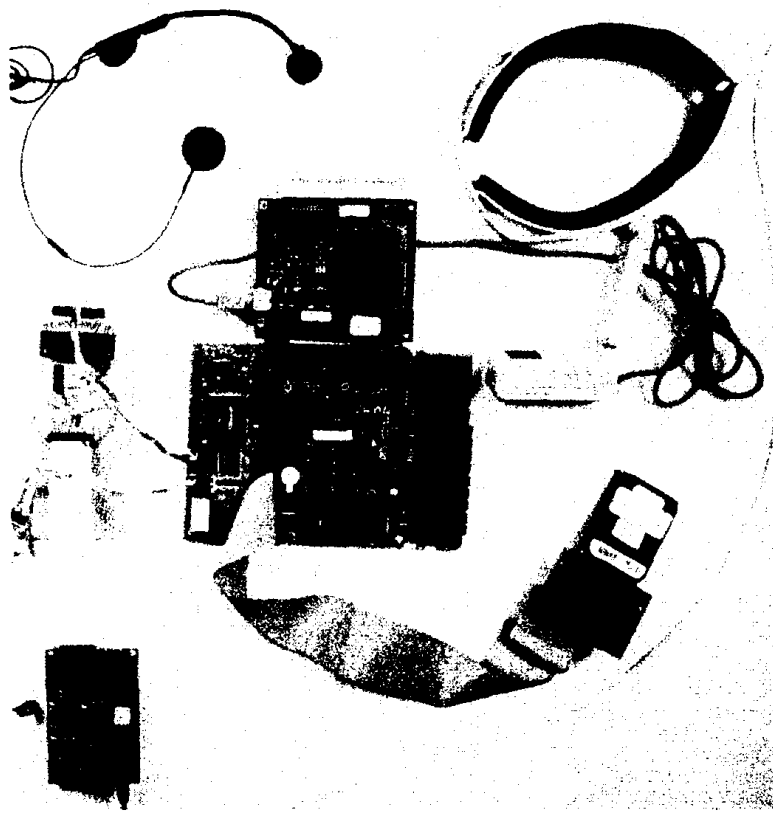

Figure 6. Navigator electronics prior to assembly. Left portion of the photo includes the microphone, GPS adapter, and GPS board. Middle portion shows the VGA card and processor board. Right side includes the Private Eye display and hard disk.

team was organized into groups, according to the subsystem break-down. During the early stages of the design process, it was recognized that the $\mathrm{HCl}$ subsystem was comprised of two independent components: speech and applications. Additional subsystems were formed to allow more concurrency in the total design process and improve organization by focusing group efforts. As the design progressed, the resource requirements of each group changed and designers were reallocated. The ability to reallocate personnel allowed for new needs to be met. For example, the applications group initially believed that their two person team would be sufficient, since the concept for the application of a campus tour seemed reasonable from the programming perspective. As the design of the campus navigation system was developed, it was clear that there were many aspects of the application that needed to be addressed. There were interface issues with speech and GPS, along with the design of the user interface. Thus, it was concluded that two designers were insufficient to complete the design on time and it was necessary to reallocate three people from the systems software group to assist in applications development. Figure 9 depicts the evolution of personnel assignments where the numbers indicate the people involved.

In parallel with the development of the various electronics and software subsystems, mock prototypes of the final system housing were built by the mechanics group to model ergonomics. As the open-air system developed, however, problems were revealed that needed to be considered by the mechanical group, e.g., constraints on buses interconnecting the various

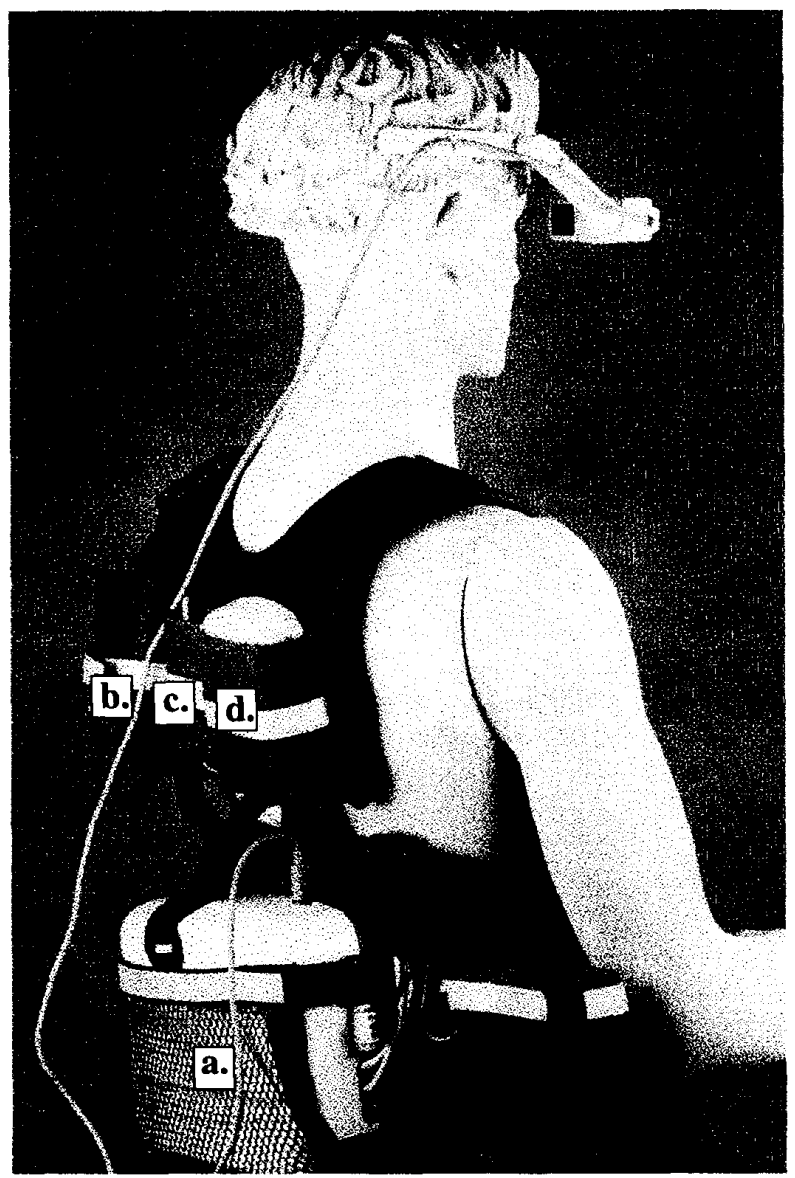

Figure 7. Navigator, a modular wearable computer:

a. Navigator housing containing the electronics

b. cellular phone

c. modem

d. GPS board

modules. Also, electrical interference and heat dissipation experiments were performed to study interactions among the electronic components of the open-air system. Therefore, the prototypes were continuously refined and updated as the openair system evolved.

\section{Communications}

The communication patterns between groups varied with the design phases since certain forms of communication were better suited to particular phases. Since alternative technologies were surveyed independently for each subsystem during the Technology survey phase, little communication was needed between design groups. Thus the main form of communication in this phase was presentations to share survey results. In the system specification phase, the first concepts for the total system were explored. Therefore, it was very important for ideas to be exchanged on proposed subsystem composition. Each group had brainstorming meetings to produce solutions for their subsystems. The results of these meetings were shared in group presentations, leading to discussions on issues related to inter- 


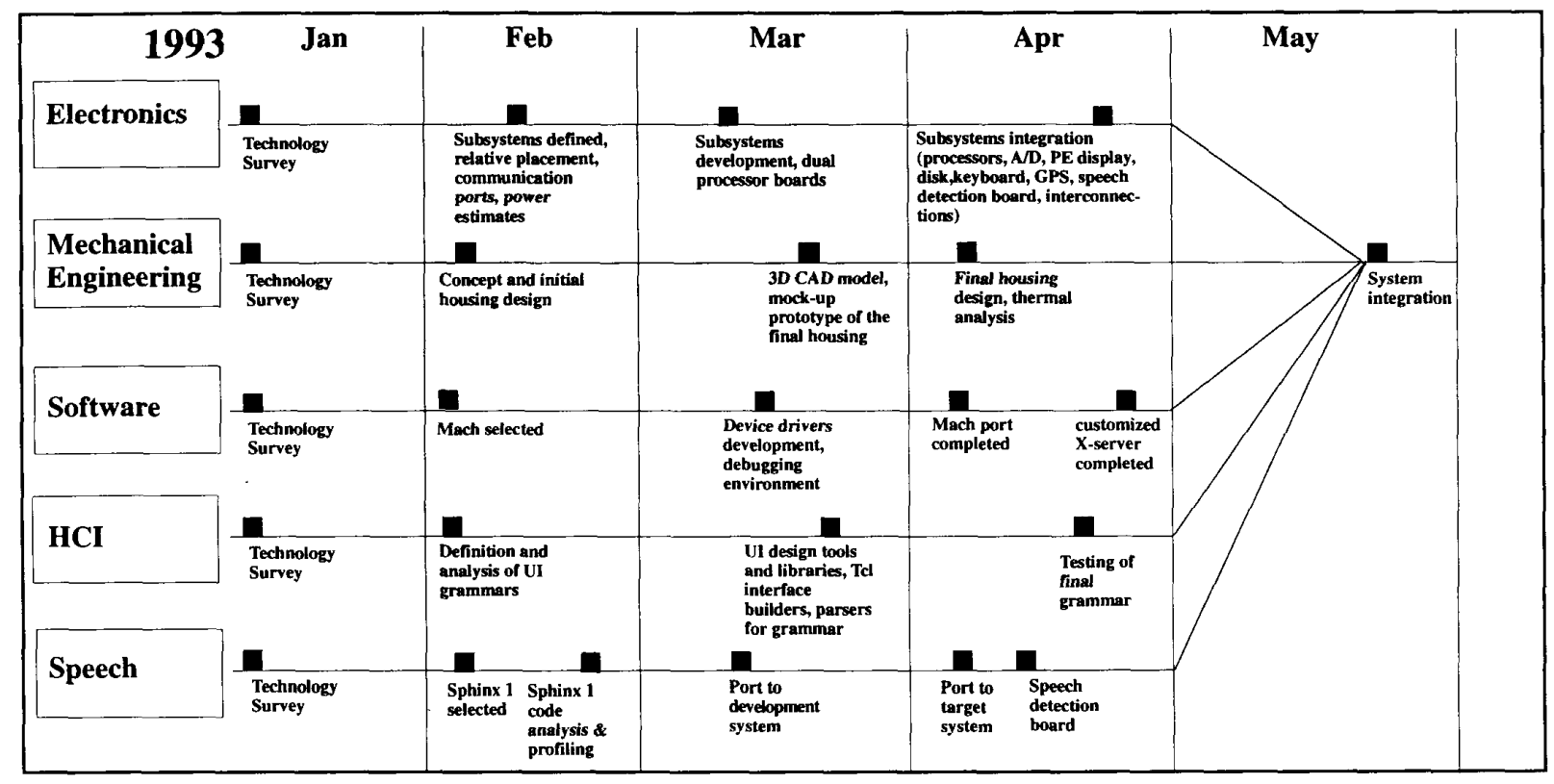

Figure 8. Time line of the Navigator project.

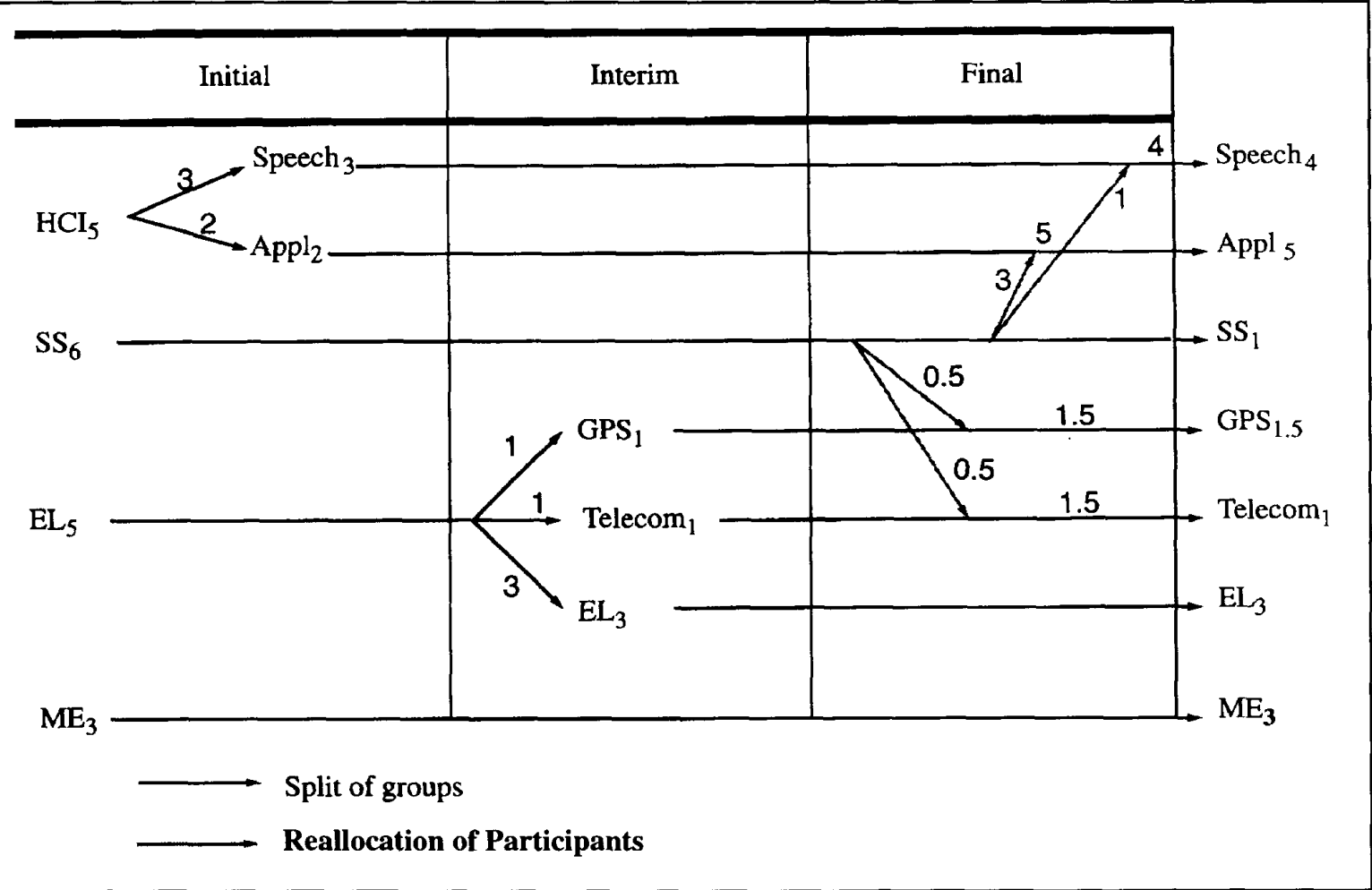

Figure 9. Organizational phases of the project, split of groups and reallocation of participants. Subscripts represent number of participants in each group. 
actions and interfaces between subsystems. As the design moved into the Subsystem Specification and Detailed Design phases, there was an increased need for inter-group communications because these stages involved design of interfaces between subsystems. To facilitate communications between the groups, liaisons were appointed, who participated in group meetings of several groups. Their function was to detect interface and interaction problems between groups, as well as to help identify hidden problems within the groups. During these phases, electronic mail became an increasingly used medium for discussing interface questions between groups.

In the implementation phase, each subsystem was built according to the detailed design done in the previous phase. Within each group, individuals were assigned by the group to complete different tasks. Thus, less time was spent in group meetings as people worked on their assigned tasks. E-mail traffic increased as individuals would encounter problems which affected many people within and outside their groups. During this phase, there were e-mail announcements of incremental progress as people completed subtasks.

In the implementation and integration phases, communication was mainly in the form of progress reviews to update the status of each group, set new milestones and explore problems. As subsystem implementation progressed, interaction between groups increased because modifications affected other groups.

The integration phase required more communication between all project members. In this phase, the subsystems were assembled into a total system. Problems were encountered as the subsystems were merged together and required the attention of affected groups. Thus, communication among all the project members was very important.

\section{Effort}

The relationship between discipline person hours and phases is shown in Table 4 . The rows correspond to the four disciplines involved in the project: Electronics, Mechanical, Human-Computer Interaction, and Software. The six phases of the project are represented in the corresponding columns. The total design effort was well balanced, where most of the time was spent in the phases of detailed design (38\%) and implementation $(24 \%)$, while the other four phases consumed almost an equal percentage of time (8-11\%). Should an inadequate effort been made in the recognition of design team inter-dependencies, a significantly larger amount of time would have been required in the integration phase for additional design iterations to correct problems.

\section{Conclusions}

This paper outlines the concurrent thermal design of the Navigator wearable computer. The generic Concurrent Design Procedure is presented and the role of the thermal designer in each design stage is described. As such, this paper represents a complete view of how thermal design integrates within a complete product design cycle. A variety of thermal analysis techniques are used, ranging from approximate Figures of Merit and one-dimensional models, to scaling analysis and, finally, to direct numerical simulations of the conjugate conduction/

\begin{tabular}{|c|c|c|c|c|c|c|}
\hline Funct. & Specech & GPS & $\begin{array}{l}\text { Telecon- } \\
\text { munication }\end{array}$ & Display & Applications & Total \\
\hline Disciplina & 105 & 177 & 181 & 653 & 342 & 1069 \\
\hline ME & 249 & 125 & 249 & 125 & 218 & 529 \\
\hline HCI & 131 & 466 & 435 & 715 & 964 & 801 \\
\hline SW & 466 & 746 & 90 & 147 & 158 & 710 \\
\hline Total & 398 & 156 & 225 & 302 & 607 & \\
\hline
\end{tabular}

Table 4. Discipline person hours per functional subsystems

convection phenomena. Each of these approaches was carefully selected to provide required information to other designers, as well as to balance time and resource constraints associated with a specific design phase. Contributions made by the thermal design group, ranged from evaluation of heat dissipation limitations of each subsystem module to providing operating temperatures of the various components.

\section{Acknowledgments}

The contributions to the design of the Navigator of the following people are acknowledged: Forrest Chamberlain, Carol Hernandez, Jason Lee, Tom Martin, Brian Nobel, Ali Reza Adl Tabatabai, and Jim Zelenka. The thermal design team would like to express gratitude to Jennifer Murphy and Nick Narisaranukul for assisting in the numerical simulations and Venkat Natarajan, for his aid in conducting the experimental measurements.

Financial support by the Advanced Research Project Agency Grant JFBI 92195 and National Science Foundation Grants EDC-8943164 and CTS-9311072 is gratefully acknowledged.

\section{References}

Akella, J., Dutoit, A., and Siewiorek, D.P., 1992, "Concurrent Engineering: A Prototyping Case Study," Proceedings of the 3rd IEEE International Workshop on Rapid System Prototyping, Research Triangle Park, N. Carolina.

Amon, C.H., 1992, "Heat Transfer Enhancement by Flow Destabilization in Electronic Chip Configurations," ASME J. of Electronic Packaging, Vol. 114, pp. 35-40.

Amon, C.H. and Mikic, B.B., 1992, "Spectral Element Simulations of Unsteady Convective Heat Transfer," Numerical Heat Transfer, Vol. 19, pp. 1-19. 
Bar-Cohen, Avram, 1992, "State-of-the-Art and Trends in the Thermal Packaging of Electronic Equipment," J. Elec. Pack., Vol. 114, pp. 257-270.

Becker, A., 1992, "High Resolution Virtual Displays," Proc. SPIE, Vol. 1664, Society of Photooptical Instrumentation Engineers, Bellingham, Wash.

Bejan, A., 1984, Convection Heat Transfer, New York: John Wiley and Sons., pp. 159-197.

Chu, R.C. and Simons, R.E., 1993, "Cooling Technology for High Performance Computers: Part 1-Design Applications," ASI Proceedings on Cooling of Electronic Systems, Cesme, Turkey, pp. 411-433.

Kraus, A.D. and Bar-Cohen, Avram, 1983, Thermal Analysis and Control of Electronic Equipment, Washington: Hemisphere Publishing Co., p. 22.

Krueger, W. and Bar-Cohen, Avram, 1992, "Thermal Characterization of a PLCC - Expanded $\mathrm{R}_{\mathrm{jc}}$ Methodology," IEEE Trans. Comps., Hybrids, Manuf. Technol., Vol. 15, No. 5, pp. 850-859.

Li, K.F., Hon, H.W., Hwang, M.J., and Reddy, R., 1989, "The Sphinx Speech Recognition System," Proceedings of the IEEE ICASSP, Glasgow, U.K.

Moffat, R.J. and Ortega, A., 1988, "Direct Air-Cooling of Electronic Components," Advances in Thermal Modeling of Electronic Components and Systems, Volume 1, eds. A. BarCohen and A. D. Kraus, New York, Hemisphere Publishing Corporation, pp. 129-265.

Nigen, J.S. and Amon, C.H., 1992, "Concurrent Thermal Designs of PCBs: Balancing Time and Accuracy Constraints," IEEE Transactions on Comps., Hybrids, and Manuf. Technol., Vol. 15, No. 5, pp. 850-859.

Nigen, J.S. and Amon, C.H., 1993, "Forced Convective Cooling Enhancement of Electronic Package Configurations through Self-Sustained Oscillatory Flows," ASME J. of Electronic Packaging, Vol. 115, pp. 356-365.

Oktay, S., 1993, "Beyond Thermal Limits in Computer Systems Cooling," ASI Proceedings on Cooling of Electronic Systems, Cesme, Turkey, pp. 184-207.

Patera, A.T., 1984, “A Spectral Element Method for Fluid Dynamics: Laminar Flow in a Channel Expansion," J. Comp. Physics, Vol. 54, pp. 468-488.

Peterson, G.P. and Ortega, A., 1990, "Thermal Control of Electronic Equipment and Devices," Advances in Heat Transfer, Vol. 20, eds. J.P. Hartnett and T.F. Irvine, Jr., San Diego: Academic Press, Inc., pp. 183-213.
Rashid, R., Teranian, A., Black, D., and Baron, R., 1989, "Mach: A System Software Kernel," COMPCON '89, IEEE Computer Society Press, Los Alamitos, CA.

Siewiorek, D.P., Smailagic, A., and Lee, J.C.Y., 1993, “An Interdisciplinary Concurrent Design Methodology as Applied to the Navigator Wearable Computer System," J. Comp. and Soft Eng., Vol. 2, No. 2.

Smailagic, A., Siewiorek, D.P., 1993, "A Case Study in Embedded Systems Design: The Vuman 2 Wearable Computer," IEEE Design and Test of Computers, Vol. 10, No. 3, pp. 56-67.

Yesilyurt, S. and Patera, A.T., 1994, "Surrogates for Numerical Simulations; Optimization of Eddy-Promoter Heat Exchangers," to appear in Computer Methods in Applied Mechanics and Engineering.

Yovanovich, M.M. and Antonetti, V.W., 1988, “Application of Thermal Contact Resistance Theory to Electronic Packages," Advances in Thermal Modeling of Electronic Components and Systems, Vol. 1, eds. Avram Bar-Cohen and Allan D. Kraus, New York: Hemisphere Publishing Co., pp. 79-128. 